\title{
Co-infections of SARS-CoV-2 with multiple common respiratory pathogens in infected patients
}

\author{
Dachuan Lin ${ }^{1 \dagger}$, Lei $\mathrm{Liu}^{2 \dagger}$, Mingxia Zhang ${ }^{2 \dagger}$, Yunlong Hu ${ }^{1}$, Qianting Yang ${ }^{2}$, Jiubiao Guo ${ }^{1}$, \\ Yongchao Guo ${ }^{1}$, Youchao Dai ${ }^{1}$, Yuzhong Xu ${ }^{3}$, Yi Cai ${ }^{1}$, Xinchun Chen ${ }^{1}$, Zheng Zhang ${ }^{2 *}$ \& \\ Kaisong Huang ${ }^{1 *}$

\begin{abstract}
${ }^{1}$ Guangdong Provincial Key Laboratory of Regional Immunity and Disease, Department of Pathology Biology, School of Medicine, Shenzhen University, Shenzhen 518060, China;

${ }^{2}$ National Clinical Research Center for Infectious Diseases, Guangdong Key Lab for Diagnosis \& Treatment of Emerging Infectious Diseases, Shenzhen Third People's Hospital, Southern University of Science and Technology, Shenzhen 518112, China;

${ }^{3}$ Department of Clinical Laboratory, Shenzhen Baoan Hospital, The Second Affiliated Hospital of Shenzhen University, Shenzhen 518071,
\end{abstract} China

Received February 17, 2020; accepted March 2, 2020; published online March 5, 2020

Citation: Lin, D., Liu, L., Zhang, M., Hu, Y., Yang, Q., Guo, J., Guo, Y., Dai, Y., Xu, Y., Cai, Y., et al. (2020). Co-infections of SARS-CoV-2 with multiple common respiratory pathogens in infected patients. Sci China Life Sci 63, 606-609. https://doi.org/10.1007/s11427-020-1668-5

\section{Dear Editor,}

In late December 2019, an outbreak of severe pneumonia caused by an unknown etiological agent was initially reported in Wuhan, China, and then quickly spread throughout China and even abroad (Zhu et al., 2020). Swift control measures and etiological investigations were conducted by the Chinese government, physicians and researchers, and by using high throughput sequencing and virological testing, the mysterious pneumonia pathogen was shown to be a novel coronavirus (SARS-CoV-2) capable of person-to-person transmission (Zhu et al., 2020). Coronaviruses are a group of non-segmented, enveloped and positive-sense RNA viruses that belong to the subfamily of Coronavirinae in the family of Coronavirdiae and order of Nidovirales. Prior to the COVID-19 outbreak, six coronaviruses have been documented to be able to infect humans. Of them, the Severe Acute Respiratory Syndrome Coronavirus (SARS-CoV)

\footnotetext{
$\uparrow$ Contributed equally to this work

*Corresponding authors (Kaisong Huang, email: kaisong@szu.edu.cn; Zheng Zhang, email: zhangzheng1975@aliyun.com)
}

and Middle East Respiratory Syndrome Coronavirus (MERS-CoV) are the most well-described coronavirus as they were responsible for two large-scale outbreaks in 2003 and 2012, with 8,096 cases infections and roughly 10\% mortality and 2,494 cases and $34.4 \%$ mortality, respectively (Cheng et al., 2007; Chan et al., 2015). Until the moment of manuscript submission, coronavirus SARS-CoV-2 has spread to over 25 countries globally and caused over 59,000 cases with 1,316 people dead already ( http://2019ncov.chinacdc.cn/2019-nCov/). Furthermore, there are still over 16,000 cases suspected in 31 provinces in China. Although these suspected patients are mostly quarantined in specialized hospitals or self-quarantined at home, a timely, effective and accurate diagnosis for those people is of pivotal importance for alleviating the already overwhelming medical system and preventions of further expansions. In this study, a fast and reliable multiplex reverse transcription-polymerase chain reaction method (multiplex rapid detection kit 2.0, Uni-MEDICA Tech, Shenzhen), which can simultaneously detect 15 respiratory tract infection pathogens including the SARS-CoV-2, was employed to screen the pathogen agents in the patients. These 15 respiratory pathogens are the 2019 
novel coronavirus (SARS-CoV-2), influenza A/B (FluA/B), coronavirus NL63 (NL63), coronavirus (229E), parainfluenza virus type 1/2/3(PIV1/2/3), adenovirus (AdV), rhinovirus (hRV), human bocavirus (hBov), coronavirus HKU1 (HKU1), coronavirus OC43(OC43), human metapneumovirus (hMPV) and respiratory syncytial virus (RSV). The sensitivity and specificity of this method have been evaluated by the kit manufacturer Uni-MEDICA Tech, and the minimum detection limits for those 15 respiratory pathogens of this kit were between 100 copies and 1,000 copies per microliter. Detailed evaluation approaches and results are available in Supplementary file 1 in Supporting Information.

According to the official instruction for diagnosing and treatment of 2019 novel coronavirus infections issued by the National Health Commission of the People's Republic of China, patients were categorized as SARS-CoV-2-suspected patients when they had an epidemiological risk and presented with two or more clinical features like fever, abnormal chest $\mathrm{CT}$ and normal or low white blood cell count or low lymphocyte count. In Shenzhen city, all SARS-CoV-2suspected patients or patients with a high possibility of SARS-CoV-2 infection were eventually transferred and admitted into the Third People's Hospital of Shenzhen. Most of these patients were admitted into this hospital in the first week after the onset of symptoms, and the first set of samples (sputum, nasal or throat swab) were collected immediately upon admission. A re-sampling was included for in-parallel testing analysis one day later after the first sampling. A total of 186 random patients' specimens collected from January 20th to February 1st in the Third People's Hospital of Shenzhen were subjected to out testing. Detailed procedures of the total nucleic acid extraction of collected specimens, subsequent TaqMan RT-PCR assays, and RT-PCR product sequencing analysis are available in Supplementary file 1 in Supporting Information.

Among the tested 186 patients, 92 (49.46\%) were males and 94 were females. The majority of patients $(145,77.96 \%)$ aged 18 to 65 years old. One hundred and forty-two (76.3\%) patients presented a fever and 124 (66.67\%) had abnormal chest CT results. Detailed individual characteristics for these patients including age, gender, days after onset, clinical feature, chest CT, and chronic diseases like diabetes, hypertension and cardiovascular diseases and blood routines are summarized in Supplementary file 2 in Supporting Information. A close examination of the medical records of these patients revealed that most of them (155 cases, $83.3 \%)$ had an epidemiologic risk including residence or traveling history to Wuhan and its nearby cities, or had a contact with patients having respiratory disease symptoms or confirmed SARS-CoV-2 infections from endemic areas in the past two weeks, indicating that most of the suspected cases were originally exported from the endemic regions into Shenzhen city.
Using this multiplex kit, we confirmed that 92 patients (49.46\%) were infected by the SARS-CoV-2 (Table 1). When compared to the testing result using the National Medical Production Administration authorized GeneoDx kit instead, we found that the total 88 individuals of SARS-CoV2 infections detected by the GeneoDx method were also all exactly identified in the multiplex approach testing (Supplementary file 3 in Supporting Information). The RT-PCR products for the four more cases of SARS-CoV-2 infections detected in the multiplex approach were further sequenceverified (Supplementary file 5 in Supporting Information). The chip-square and Fisher's exact test were employed to compare the difference between these two methods, and no statistically significant difference was detected for them (Supplementary file 3 in Supporting Information). In the linear regression analysis, the calibration equation is $y=1.0146 x-1.8086$ with the correlation coefficient of 0.7866 (Supplementary file 3 in Supporting Information). Of the 94 cases $(50.54 \%)$ with a negative reaction for the SARS-CoV2, the multiple panel test kit detected the presence of one or more of hRV, RSV, AdV, FluA, FluB , PIV2, PIV3, HKU1 and hMPV virus in 18 patients $(9.7 \%)$, with five patients presenting three viruses in their respiratory tracts (Supplementary file 4 in Supporting Information). These infections were verified by sequencing the RT-PCR products as well (Supplementary file 5 in Supporting Information). These results together suggested that this multiplex panel approach can not only serve as a sensitive and reliable tool for identifications of SARS-CoV-2 infection in the community but also help the physician to remove the common respiratory pathogen infection cases from the suspected or probable SARS-CoV-2 infection categories. This removal of non-infected cases will help to relieve the current pressures of hospitalizations and isolations for those patients, and further alleviate the following requirements for epidemiologic investigation and contact tracing, thus saving the current extremely limited medical and human resources.

Intriguingly, in the 92 SARS-CoV-2 (49.46\%) positive patients, the common respiratory viruses RSV, hRV, hMPV, PIV2 and HKU1 were also simultaneously detected in six patients $(3.2 \%)$ respectively, of which four patients $(2.2 \%)$ were positive for at least two detected viruses (Supplementary file 4 in Supporting Information). The co-infections in these six patients were further verified in our parallel testing using a second-day sampling from the same patients. As far as we know, this is the first solid evidence for the co-infection of SARS-CoV-2 with other respiratory viruses in patients although it remains conceivable. The implications of these co-infections in patients deserve further studies particularly from a perspective of virus evolution and patients' clinical symptoms.

A total of $76(40.86 \%)$ cases were negative for all pathogens in our test. We speculate that this is probably due to the 
Table 1 Comparisons of clinical symptoms, blood routine test results and epidemiologic risks between different infection categories

\begin{tabular}{|c|c|c|c|c|c|c|c|c|c|c|c|}
\hline Infection type & Cases & Fever & Cough & Dyspnea & Chest CT & Leukopenia & $\begin{array}{l}\text { Lymphocyte } \\
\text { normal }\end{array}$ & Lymphopenia & $\begin{array}{c}\text { No epidemiologic } \\
\text { risks }^{\text {a) }}\end{array}$ & $\begin{array}{l}\text { Patients with } \\
\text { epidemiologic } \\
\text { risks and minor } \\
\text { signs }\end{array}$ & $\begin{array}{l}\text { SARS-CoV-2- } \\
\text { suspected } \\
\text { patients }\end{array}$ \\
\hline Single SARS-CoV-2 & 86 & 61 & 40 & 6 & 55 & 9 & 76 & 13 & 8 & 15 & 63 \\
\hline $\begin{array}{c}\text { Co-infections of } \\
\text { SARS-CoV-2 and RDVs) }\end{array}$ & 6 & 1 & 1 & 0 & 4 & 2 & 3 & 2 & 1 & 1 & 4 \\
\hline Other RDVs positive & 18 & 15 & 13 & 1 & 12 & 1 & 15 & 5 & 5 & 2 & 11 \\
\hline All negative & 76 & 60 & 42 & 4 & 52 & 4 & 68 & 12 & 17 & 6 & 53 \\
\hline
\end{tabular}

a) Those patients are diagnosed as SARS-CoV-2-suspected patients when they have one epidemiological risk and two clinical features below according to the official instruction for diagnosing and treatment of 2019 novel coronavirus infections issued by the National Health Commission of the People's Republic of China. Epidemiologic risks include three conditions below: (1) Patients have been resident or traveled to Wuhan and its nearby cities in Hubei province, China in the past two weeks. (2) Patients have contact with people who presented a fever or respiratory disease symptoms and had a travel history to Wuhan and its nearby cities in the past two weeks. (3) Patients have contacted people with confirmed SARS-CoV-2 infection in the past two weeks. Clinical features: (1) Fever, temperature over 37.3. (2) The patient's chest CT scan presents a pneumonia symptom including apparent extrapulmonary band, the ground glass opacity, or sub-segmental areas of consolidation. (3) Normal or decreased white blood cell counts or reduced lymphocyte counts in blood routine tests at the earlier stage of the disease. b) RDVs indicate the other 14 respiratory tract disease viruses excluding the SARS-CoV-2 that were simultaneously screened by our multiplex kit.

presence of other pathogen infections like the mycoplasma pneumonia or other bacteria, which were generally rampant during the current season (Waites and Talkington, 2004; Zivich et al., 2018). Additionally, the intrinsic limitation of the sensitivity of this method for the different respiratory viruses and low load of virus in upper respiratory specimens may also partially contribute to the negative results. Both aspects merit further investigations.

A retrospective analysis for clinical symptoms, blood routine test results and the epidemiologic risk in these different infection categories was conducted and compared (Table 1). Eleven people (5.9\%), previously classified as SARS-CoV-2-suspected patients, based on the official instruction for diagnosing and treatment of 2019 novel coronavirus infections issued by the National Health Commission of the People's Republic of China, were found to be infected by other common respiratory viruses. Furthermore, among the 131 (70.4\%) patients who fulfilled all of the clinical signs and epidemiologic risk of SARS-CoV-2suspected cases, 64 (34.4\%) were negative for the SARSCoV-2 virus. Notably, $15(8 \%)$ patients, previously not grouped with SARS-CoV-2-suspected patients due to lack of apparent clinical features (despite the presence of epidemiological risks), were confirmed to be infected by the SARS-CoV-2. These results compellingly indicate that a careful contact tracing for confirmed patients and strict quarantine strategy for potential SARS-CoV-2 patients with possible exposure to the virus are still of pivotal importance for controlling the COVID-19 expansions.

In summary, our studies documented for the first time the co-infection of the SARS-CoV-2 with multiple common respiratory pathogens taking place in the community. In addition, SARS-CoV-2 infections were also discovered in individuals who were not SARS-CoV-2-suspected patients due to lack of apparent clinical symptoms, indicating that a careful contact tracing and strict quarantine strategy are still crucial for controlling the SARS-CoV-2 expansions.

Compliance and ethics The author(s) declare that they have no conflict of interest.

Acknowledgements This work was supported by Guangdong Provincial Science and Technology Program (2019b030301009), the National Natural Science Foundation of China (81802060), and the start-up funding of Shenzhen University and the National Science and Technology Major Project (2017ZX10201301).

\section{References}

Chan, J.F.W., Lau, S.K.P., To, K.K.W., Cheng, V.C.C., Woo, P.C.Y., and Yuen, K.Y. (2015). Middle East respiratory syndrome coronavirus: another zoonotic betacoronavirus causing SARS-like disease. Clin Microbiol Rev 28, 465-522.

Cheng, V.C.C., Lau, S.K.P., Woo, P.C.Y., and Yuen, K.Y. (2007). Severe acute respiratory syndrome coronavirus as an agent of emerging and reemerging infection. Clin Microbiol Rev 20, 660-694.

Waites, K.B., and Talkington, D.F. (2004). Mycoplasma pneumoniae and its role as a human pathogen. Clin Microbiol Rev 17, 697-728.

Zhu, N., Zhang, D., Wang, W., Li, X., Yang, B., Song, J., Zhao, X., Huang, B., Shi, W., Lu, R., et al. (2020). A novel coronavirus from patients with pneumonia in China, 2019. N Engl J Med 382, 727-733.

Zivich, P.N., Grabenstein, J.D., Becker-Dreps, S.I., and Weber, D.J. (2018). Streptococcus pneumoniae outbreaks and implications for transmission and control: a systematic review. Pneumonia 10, 11. 


\section{SUPPORTING INFORMATION}

Supplementary file 1 Materials and methods and approaches evaluation results.

Supplementary file 2 Individual characteristics for these patients including age, gender, days after onset, and clinical features.

Supplementary file 3 Comparison between the GeneoDX (1) and Uni-MEDICA (2) method for detecting 2019 novel coronavirus in patients.

Supplementary file 4 Co-infection of SARS-CoV-2 with other pathogens or merely other pathogen infections.

Supplementary file 5 Sequence for 4 more SARS-CoV-2 and other respiratory positive cases infections.

The supporting information is available online at http://life.scichina.com and https://link.springer.com. The supporting materials are published as submitted, without typesetting or editing. The responsibility for scientific accuracy and content remains entirely with the authors. 